\title{
ANÁLISE CONTEXTUAL DA TEORIA DAS REPRESENTAÇÕES SOCIAIS NA PERSPECTIVA DA PESQUISA QUALITATIVA EM ENFERMAGEM
}

\section{CONTEXTUAL ANALYSIS OF THE SOCIAL REPRESENTATIONS THEORY IN THE PERSPECTIVE OF QUALITATIVE RESEARCH IN NURSING}

\section{ANÁLISIS CONTEXTUAL DE LA TEORÍA DE LAS REPRESENTACIONES SOCIALES EN LA PERSPECTIVA DE LA INVESTIGACIÓN CUALITATIVA EN ENFERMERÍA}

Mônica Gisele Costa Pinheiro ${ }^{1}$, lellen Dantas Campos Verdes Rodrigues ${ }^{2}$, Gustavo Ávila Dias, Emanuella de Castro Marcolino ${ }^{3}$, Bruna Raquel da Silva Gomes ${ }^{4}$, Francisco Arnoldo Nunes de Miranda ${ }^{5}$.

\begin{abstract}
RESUMO
Objetivo: analisar os aspectos contextuais que envolvem a Teoria das Representações Sociais, na perspectiva da pesquisa qualitativa em enfermagem. Método: ensaio analítico, fundamentado no referencial teórico da análise contextual, como fonte de significado e conhecimento, no qual o contexto é compreendido, em quatro níveis de camadas distintas, mas que mantém relações entre si. Resultados: o contexto imediato aborda a utilização da Teoria das Representações Sociais nas pesquisas em enfermagem; o contexto específico discorre sobre a pesquisa qualitativa na área da enfermagem; os aspectos subjetivos inerentes à Teoria das Representações Sociais nas pesquisas qualitativas em enfermagem estão dispostos no contexto geral. Cabe ao metacontexto traçar considerações sobre a amplitude que encerra as pesquisas, em enfermagem, de modo geral. Conclusão: a discussão exposta, no presente estudo, ampliou a compreensão dos diversos contextos que envolvem a Teoria das Representações Sociais, na perspectiva da pesquisa qualitativa em enfermagem.
\end{abstract}

Descritores: Teoria social; Pesquisa qualitativa; Pesquisa em enfermagem.

\begin{abstract}
Objective: the objective of this study is to analyze the contextual aspects that involve the Theory of Social Representations in the perspective of qualitative research in nursing. Method: It is an analytical essay based on the theoretical framework of contextual analysis, as a source of meaning and knowledge, in which the context is understood at four levels of distinct layers, but they maintain relations with each other. Results: The immediate context discusses the use of Social Representations Theory in nursing research. The specific context discusses the qualitative research in the field of nursing. The subjective aspects inherent to the Social Representations Theory in qualitative research in Nursing are arranged in the general context. It is the responsibility of the metacontext plot considerations about the extent that closes the nursing research in general. Conclusion: The discussion presented in the present study expanded the understanding of the different contexts that involve the Theory of Social Representations in the perspective of qualitative research in nursing.
\end{abstract}

Descriptors: Social theory; Qualitative research; Nursing research.

\section{RESUMEN}

Objetivo: analizar los aspectos contextuales que envuelven la Teoría de las Representaciones Sociales en la perspectiva de la investigación cualitativa en enfermería. Método: ensayo analítico fundamentado en el referencial teórico del análisis contextual, como fuente de significado y de conocimiento, en el cual el contexto es comprendido en cuatro niveles de capas distintas, pero que mantiene relaciones entre sí. Resultados: el contexto inmediato aborda la utilización de la Teoría de las Representaciones Sociales en las investigaciones en enfermería; el contexto específico discurre sobre la investigación cualitativa en el área de la enfermería; los aspectos subjetivos inherentes a la Teoría de las Representaciones Sociales en las investigaciones cualitativas en enfermería están dispuestos en el contexto general. El metacontexto aborda las investigaciones en enfermería, de modo general. Conclusión: la discusión expuesta en el presente estudio amplió la comprensión de los diversos contextos que involucran la Teoría de las Representaciones Sociales en la perspectiva de la investigación cualitativa en enfermería.

Descriptores: Teoría social; Investigación cualitativa; Investigación en enfermería.

${ }^{1}$ Graduada em Enfermagem. Doutoranda pelo Programa de Pós-Graduação em Enfermagem da Universidade Federal do Rio Grande do Norte. ${ }^{2}$ Graduada em Enfermagem. Doutoranda em Enfermagem pela Universidade Federal de Sergipe. ${ }^{3}$ Graduado em Enfermagem. Mestre em antropologia social pela Universidade Federal de Sergipe. ${ }^{4}$ Graduanda em Enfermagem pela Universidade Federal do Rio Grande do Norte. Graduado em Enfermagem. Doutor em enfermagem.

Como citar este artigo:

Pinheiro MGC, Rodrigues IDCV, Dias GA, et al. Análise contextual da teoria das representações sociais na perspectiva da pesquisa qualitativa em enfermagem. Revista de Enfermagem do Centro Oeste Mineiro. 2019;8:e2722. [Access 


\section{INTRODUÇÃO}

A enfermagem constitui-se em teoria e prática de múltiplas formas de conhecimento, oriundas de saberes científicos diversos, a exemplo das ciências sociais, comportamentais e biológicas ${ }^{(1)}$. Como ofício, carrega consigo características peculiares e habilidades inerentes à descrição, explicação e prescrição do cuidado diante da complexidade e diversidade do objeto. Tais características são evidenciadas por estudos realizados sobre os fenômenos em saúde, sobretudo, os que envolvem a relação enfermeiro-cliente ${ }^{(2)}$.

Para atender às demandas de saúde e criar meios eficazes de intervir sobre elas, é necessário que a enfermagem produza conhecimento de forma ampla e plural ${ }^{(3)}$. Diferentes paradigmas que norteiam as pesquisas em enfermagem estão pautados, na utilização de rigorosos métodos, com o intuito de produzir cientificamente diferentes formas de cuidado. Para além das pesquisas quantitativas, influenciadas predominantemente pelo empirismo lógico do método cartesiano, destacam-se, em outra perspectiva, os estudos de cunho qualitativo, que se diferenciam por enfocar a subjetividade da experiência humana ${ }^{(1)}$.

A pesquisa qualitativa é estimada, em diferentes áreas do conhecimento, tanto nacionais quanto internacionais ${ }^{(4)}$. $\mathrm{Na}$ área $\mathrm{da}$ saúde, sua importância está comumente atrelada à dimensão subjetiva dos processos e significados atribuídos pelos sujeitos, a partir de experiências de saúde e doença e na elaboração de estudos originais, que utilizam abordagens inovadoras proporcionadas pelas especificidades teóricometodológicas ${ }^{(5)}$.

Além disso, trazem subsídios para fortalecer a atenção em saúde, à medida que incorporam o significado e a intencionalidade dos diferentes atores envolvidos no processo, bem como a estruturação dos atos, das relações e estruturas sociais ${ }^{(6)}$. A pesquisa qualitativa pode estar ainda articulada à teoria das representações sociais, ao se debruçar sobre elementos subjetivos, afetivos e mentais, assim como à estruturação das relações sociais que afetam as representações de dada realidade sobre a qual se deseja conhecer para intervir ${ }^{(7)}$.

Pesquisas evidenciam que 0 termo "representações sociais" teve origem, em 1961, sendo utilizado primeiramente por Serge Moscovici. O autor trabalhou as representações sociais no estudo "Psychanalyse: son image et son public", no qual abordava a representação social da psicanálise, considerando-a não apenas como conceito, mas como fenômeno. Ele as define como aglomerado sistemático de conhecimentos práticos do cotidiano, os quais são formulados coletivamente, utilizando-se dos problemas e obstáculos impostos por fenômenos entendidos como desconhecidos e não familiares que desafiam os indivíduos a modificar o próprio posicionamento e as formas de pensar, sentir e agir $^{(8)}$.

Ao considerar a Teoria das Representações Sociais (TRS), como elemento que tem contribuído com a discussão de aspectos teóricos e metodológicos dentro do campo que envolve as pesquisas em saúde ${ }^{(7)}$, este artigo objetivou analisar os aspectos contextuais, que envolvem a Teoria das Representações Sociais, na perspectiva da pesquisa qualitativa em enfermagem.

A utilização de estratégias metodológicas em pesquisas qualitativas possibilita melhor compreensão de fenômenos estudados. Isso é fundamental, quando se considera que os acontecimentos inerentes à disciplina da enfermagem são tidos como complexos, requerendo abordagens diversificadas, com combinações de diferentes possibilidades metodológicas de investigação.

\section{MÉTODOS}

O presente artigo refere-se a um ensaio analítico, fundamentado no referencial teórico da análise contextual, como fonte de significado e conhecimento. Utilizou-se da modalidade de contexto, a fim de compreender a amplitude do significado do fenômeno em estudo ${ }^{(9)}$, isto é, a produção de conhecimento, no âmbito dos cuidados de enfermagem, envolvendo pesquisa qualitativa e as possíveis relações com a teoria das representações sociais.

O contexto é compreendido como a relação existente entre o fenômeno e as diversas ocasiões em que ocorre. O conhecimento dos elementos que envolvem determinado fenômeno é imprescindível, para entender o todo de uma situação, descobrindo significados, por meio de relação mútua, proposital e sistemática ${ }^{(9)}$.

A discussão do fenômeno, a partir de diferentes contextos, amplia a sua compreensão, atribuindo-lhe significado ao relacionar o conhecimento inerente a cada um dos múltiplos contextos que envolvem a temática abordada ${ }^{(9)}$.

De acordo com o referencial utilizado para a análise, o contexto é compreendido, em quatro 
níveis de camadas distintas, mas que mantém relações entre si: o contexto imediato, cujo foco é o presente, com descrição do fenômeno em si; o contexto específico, cuja principal característica é o conhecimento individualizado e que engloba aspectos relevantes da presente situação; o contexto geral, no qual são descritos aspectos que envolvem elementos subjetivos ao fenômeno em estudo; e o metacontexto, que reúne aspectos macro do objeto de estudo ${ }^{(9)}$.

\section{RESULTADOS E DISCUSSÃO}

Embora os contextos apresentem pontos de intersecção entre $\mathrm{si}$, as camadas serão apresentadas separadamente, seguindo a divisão em quatro partes, conforme proposto pelo referencial teórico ${ }^{(9)}$, a fim de facilitar didaticamente a compreensão. Neste propósito, o contexto imediato aborda a utilização da TRS nas pesquisas em enfermagem. No que concerne ao contexto específico, envolve questões relacionadas à pesquisa qualitativa na área da enfermagem. Os aspectos subjetivos inerentes à TRS, nas pesquisas qualitativas em enfermagem, estão dispostos no contexto geral. Cabe ao metacontexto traçar considerações sobre a amplitude que encerra as pesquisas em enfermagem, de modo geral.

\section{Contexto imediato: utilização da teoria das representações sociais nas pesquisas em enfermagem}

As críticas ao modelo positivista, presentes nas demais teorias, levou Moscovici a adentrar o campo das Representações Sociais e utilizá-las como metodologia científica. Hoje, as produções fundamentadas na TRS, incluindo aquelas desenvolvidas no campo da saúde, encontram-se marcadas pela vitalidade, transversalidade e complexidade dessa vertente teóricometodológica ${ }^{(10-11)}$.

As pesquisas construídas sob o fundamento da TRS buscam, portanto, aliar de forma geral, a problematização conceitual quanto à sofisticação metodológica. No que remete à produção de conhecimento no campo da enfermagem, tais pesquisas têm favorecido a expansão das fontes de estudo.

Em geral, pode-se inferir que há ampliação do objeto, ao mesmo tempo em que há desconstrução de visão deturpada, em que a TRS é tida como algo simples, superficial e que reflete mera e superficialmente as desigualdades sociais $^{(12)}$.
No Brasil, o primeiro grupo de pesquisa sobre Representações Sociais, cadastrado no Conselho Nacional de Desenvolvimento Científico e Tecnológico (CNPq), teve origem em 1967 e as pesquisas versavam acerca dos estudos na área da Física. Percebe-se crescimento e evolução contínua e linear dos grupos de pesquisas com representações sociais e das produções envolvendo a temática, sendo 2010 o ano de maior expansão desse conhecimento, ocorrendo com maior frequência nas ciências sociais, seguida pelas ciências da saúde. Dentre as áreas que compõem as ciências da saúde, a enfermagem aparece no ranking como a terceira com maior número de produções no contexto das representações sociais ${ }^{(13)}$.

Desde a década de 1970, com a expansão dos programas de pós-graduação em enfermagem, houve aumento substancial nas publicações que utilizam a Teoria das Representações Sociais como referencial teórico ou metodológico. Esse fato reflete a autonomia da produção de conhecimento e ampliação da enfermagem como ciência e profissão, em consequência da aquisição e legitimação de um saber próprio ${ }^{(14)}$. As possibilidades inerentes ao uso da TRS conferem liberdade ao pesquisador de enfermagem tanto na escolha do método de coleta dos dados, como em sua análise. Trata-se de fonte diversificada de dados quantitativos e qualitativos a respeito dos fenômenos investigados.

\section{Contexto específico: pesquisa qualitativa na área da enfermagem}

A pesquisa qualitativa, de modo geral, intenta compreender as relações, representações, crenças e percepções, inerentes às interpretações individuais e subjetivas que 0 sujeito faz sobre o modo como vivem ou constroem suas vidas e a $\mathrm{si}^{(6)}$. Tais pesquisas têm como objeto principal a pessoa, de tal modo que se torna pertinente a investigação dos significados, das percepções, dos padrões e das experiências relacionadas ao contexto do humano.

Pode-se afirmar que a TRS, incorporada aos estudos qualitativos, tem como característica a valorização da experiência individual em contexto coletivo. Constitui-se, assim, em valoroso e distinto recurso às pesquisas de saúde e enfermagem, sobretudo, em situações nas quais se interpelam experiências e significados particulares ${ }^{(15)}$. Realizá-las, todavia, consiste em 
desafio ao pesquisador, requer dedicação, empenho, compromisso, recursos, capacidade de abstração e reflexão teórica, bem como competência analítica e compromisso ético na realização da função social ${ }^{(16)}$.

A pesquisa qualitativa manifesta-se holisticamente, sendo o indivíduo e a subjetividade o foco de investigação. Assim como na TRS, o objeto da pesquisa qualitativa não prescinde de contexto e especificidades do objeto. É, ainda, tida como "naturalista", em função da ruptura com qualquer engessamento proposto pelo pesquisador ${ }^{(17)}$.

$\mathrm{Na}$ enfermagem, a pesquisa qualitativa objetiva a valorização dos sujeitos, busca dar voz ao indivíduo, permite a livre expressão das visões de mundo. Para tanto, o pesquisador deve estar atento aos relatos dos sujeitos, utilizando-se da escuta ativa como mecanismo para ouvir e entender a realidade expressa pela voz do indivíduo, sem tornar os dados empíricos, visto que se fundamentam em métodos científicos, com base em evidências ${ }^{(18)}$.

Contexto geral: aspectos subjetivos inerentes à teoria das representações sociais nas pesquisas qualitativas em enfermagem

Os achados da pesquisa qualitativa dão respostas às questões centradas na experiência social e ampliam o significado da vida humana. São bastante usados, uma vez que valores humanos, culturais e relacionamentos não podem ser mensurados ou descritos, mediante o uso de métodos de pesquisa quantitativa ${ }^{(1)}$.

O elemento ético das pesquisas qualitativas é dado pelo caráter humanístico, inter-relacional e empático. Na área de saúde, tais pesquisas subsidiam a compreensão que usuários, profissionais de saúde e gestores têm diante dos mais diversos elementos envoltos pela temática saúde, como a organização do sistema e dos serviços oferecidos, adoecimento, morte, saúde e as diversas representações sobre saúde ${ }^{(6)}$.

À medida que abordagens metodológicas de cunho qualitativo dão ênfase à perspectiva do paciente e de usuários de saúde, ganham evidência nas pesquisas de enfermagem. A construção de significados possibilita ao enfermeiro pesquisador a compreensão de questões relacionadas ao processo saúde e doença, bem como dos sentimentos, valores e das crenças diante de situação de saúde, mediante a sensibilidade, ética e capacidade de reflexão ${ }^{(6,16)}$.
Desenvolver ações de cuidado, com foco na singularidade dos sujeitos, requer da enfermagem, enquanto profissão, aporte teóricoprático embasado no conhecimento científico. Torna-se relevante, diante desse conhecimento, as dimensões constitutivas do sujeito que vão além de corpo biológico, isto é, compreendem fatores individuais, socioambientais e culturais que interferem e que são determinantes na da saúde da população(2).

Nesse ínterim, a TRS ganha destaque, na área da saúde, ao abordar os fenômenos de natureza sociocultural, relativos à vida social dos grupos, expressa por valores e regras sociais que regem as relações entre os indivíduos e entre eles e o mundo, orientando e organizando comportamentos em sociedade ${ }^{(7,18)}$.

A TRS valoriza a dimensão subjetiva do indivíduo, a qual influencia nas práticas sociais, atitudes e condutas. Além disso, focaliza a atenção no conhecimento dos participantes, enquanto conhecimento importante para se compreender o próprio cotidiano ${ }^{(19-20)}$.

\section{Metacontexto: diversas fases da pesquisa em enfermagem}

O movimento da Enfermagem Baseada em Evidência impulsionou crescente avanço na área da pesquisa, denotando na profissão a necessidade de os profissionais consumirem e produzirem conhecimentos próprios, inerentes à prática, em diferentes contextos da assistência, gerência ou educação. Estudos com rigor metodológico e corpo teórico consistente têm sido destaque, nas produções da área, em especial, em revisões sistemáticas da literatura, que se apresentam como padrão-ouro de evidência científica, fornecendo conhecimentos aprofundados e amplos acerca do fenômeno em estudo, o que permite ao profissional, ao utilizála, decidir pela melhor conduta a ser tomada ${ }^{(21)}$.

A expansão de Grupos de Pesquisas e Programas de Pós-Graduação em Enfermagem também contribuiu para o desenvolvimento de pesquisas científicas que dialogam com as mais diversas áreas da saúde. Esse avanço nas pesquisas foi possível pela existência de programas engajados com o avanço da ciência de enfermagem brasileira, reflexo de universidades, com oferta qualificada de mestres e doutores na área, bem como o aumento de grupos de pesquisa em enfermagem. A contribuição dessa revolução científica, na enfermagem, expressa-se, por meio de benefícios aos setores equânimes da 
sociedade, ou seja, a enfermagem brasileira avançou na busca por identificar e propor soluções aos problemas de saúde emergentes no Brasil $^{(22)}$.

Neste contexto, os órgãos de fomento à pesquisa e as políticas de incentivo à produção científica vislumbram ampliar o crescimento das publicações no país. Para tal, investem em várias interfaces, realizando financiamento a grupos de pesquisas e programas de pós-graduação, financiando projetos de pesquisadores, além da cobrança, por meio da avaliação da produtividade dos próprios programas, que consiste em método rigoroso de incentivo à produção acadêmica ${ }^{(23)}$.

No entanto enfatiza-se a importância de a Enfermagem abraçar ainda mais a pesquisa como ferramenta de transformação da práxis, assim, será possível repensar os modos de fazer, de pesquisar e de educar, acarretando avanços e transformações, nos currículos dos cursos de Graduação e Pós-Graduação do país, com consequente melhoria no trabalho de enfermagem e na saúde brasileira ${ }^{(24)}$.

\section{CONSIDERAÇÕES FINAIS}

A discussão exposta no presente estudo ampliou a compreensão dos diversos contextos que envolvem a TRS, na perspectiva da pesquisa qualitativa em enfermagem, permitindo compartilhamento e aumentando as possibilidades, para o desenvolvimento de pesquisas com valorização da subjetividade dos indivíduos, para a aplicação no cuidado de enfermagem.

Enaltece-se que cada uma das camadas interativas do contexto se distingue entre si, mediante a abrangência do conteúdo que abarcam, indo de aspectos mais específicos para os mais amplos e universais, possibilitando análise e interpretação de forma abrangente dos resultados do estudo.

Ao seguir a perspectiva de tais considerações, averiguou-se que, dentre os diversos enfoques teóricos e metodológicos, presentes nas pesquisas em enfermagem, a TRS merece destaque, pois possibilitar ao pesquisador a compreensão das atitudes, dos comportamentos e significados compartilhados por determinado grupo social. Portanto o conhecimento das representações sociais favorece a identificação de práticas estabelecidas, em certo grupo, gerando intervenções de enfermagem mais específicas e, em consequência, mais eficientes, por respeitarem as características de cada grupamento social.

Portanto, ao interligar e analisar os níveis de interação contextual, acredita-se que a utilização da TRS, associada às pesquisas qualitativas em enfermagem, possa motivar enfermeiros a prestar assistência de forma ampla, ao envolver o indivíduo, bem como particularidades e aspectos específicos imersos no fenômeno estudado.

Ademais, o estudo apresentou como limitação o enfoque subjetivista-compreensivista, inerente ao tipo de pesquisa, capaz de delimitar o potencial de reprodução, mas que atrelado ao caráter crítico-reflexivo e ao poder de abrangência da teoria utilizada, pode ser minimizado e garantir-lhe o caráter científico.

\section{REFERÊNCIAS}

1- Silva FS, Pinheiro MGC, Moreira BGL, Simpson $\mathrm{CA}$, Vitor AF, Lira ALBC. Contribuições da história oral para $O$ cuidado de enfermagem: Revisão integrativa. Revista de Enfermagem Centro-Oeste Mineiro. 2014;4(2):1204-13. DOI: 10.19175/recom.v0i0.658

2- Ferreira MA. Teoria das Representações Sociais e contribuições para as pesquisas do cuidado em saúde e de enfermagem. Esc. Anna Nery 2016;20(2):214-9. DOI: 10.5935/14148145.20160028

3- Soares CB, Hoga LAK, Peduzzi M, Sangaleti C, Yonekura T, Silva DRAD. Revisão integrativa: Conceitos e métodos utilizados na enfermagem. Rev Esc Enferm USP 2014;48(2):335-45. DOI: 10.1590/S0080-6234201400002000020

4- Knauth DR, Leal AF. A expansão das Ciências Sociais na saúde coletiva: Usos e abusos da pesquisa qualitativa. Interface 2014;18(50):45767. DOI: $10.1590 / 1807-57622014.0274$

5- Gomes MHA, Martin D, Silveira C. Comentários pertinentes sobre usos de metodologias qualitativas em saúde coletiva. Interface 2014;50(18):469-77. DOI: $\quad \underline{10.1590 / 1807-}$ $\underline{57622014.0271}$

6- Minayo MCS, Guerriero ICZ. Reflexividade como éthos da pesquisa qualitativa. Ciência Saúde Coletiva 2014;19(4):1103-12. DOI: 10.1590/1413-81232014194.18912013

7- Lefevre F, Lefevre AMC. Discurso do sujeito coletivo: Representações sociais e intervenções comunicativas. Texto Contexto-Enferm. 2014;23(2):502-7. DOI: $\quad$ 10.1590/0104$\underline{07072014000000014}$ 
8- Moscovici S. Representações sociais: Investigações em psicologia social. Petrópolis: Vozes; 2013.

9- Hinds PS, Chaves DE, Cypress SM. Context as a source of meaning and understanding. Qual Health Res. 1992;2(1):61-74. DOI: 10.1177/104973239200200105

10- Medina Filho AL. Importância das imagens na metodologia de pesquisa em psicologia social. Psicol Soc. 2013 [citado em 15 novembro 2018]; 25(2):263-71. Disponível em: http://www.scielo.br/scielo.php?script=sci arttext \&pid=S0102-71822013000200003\&lng=pt\&nrm=iso 11- Silva CB, Carmo GT, Silva AMC. Interdisciplinaridade e teoria das representações sociais: Lacônicas notas. Tematica 2014 [citado em 20 out 2018]; 10(12):32-41. Available in: http://periodicos.ufpb.br/ojs/index.php/tematica Larticle/view/22134

12- Nascimento ARA, Trindade ZA, Veloso FGC, Nogueira EL, Vieira GT, Cezar VCF. Representações sociais, gênero e saúde no Brasil: Uma revisão bibliográfica. Tempus 2012;6(3):26578. DOI: $\underline{10.18569 / \text { tempus.v6i3.1168 }}$

13- Martins AM, Carvalho CAS, Antunes-Rocha MI. Pesquisa em representações sociais no Brasil: Cartografia dos grupos registrados no CNPq. Psicol Teor Prát. 2014;16(1):104-14. DOI: 10.15348/1980-6906/psicologia.v16n1p104-114

14- Alves MDS, Barroso MGT, Oriá MOB, Teixeira MCTV. A Teoria das Representações Sociais na pós-graduação em enfermagem: A realidade brasileira. Rev Enferm UERJ 2005;13(3):331-9. DOI: $10.1590 /$ S0034-71672011000500022

15- Melo LP, Jensen R, Oliveira ALO, Jaeger $M$, Salim NR, Musselli AR. Pesquisa qualitativa como estratégia de ensino em humanização do cuidado de enfermagem. Rev Eletr Gestão Saúde 2014 [citado em 7 out 2018]; 5(3):1130-8. Available in: http://periodicos.unb.br/index.php/rgs/article/vi ew/22704

16- Gonçalves $\mathrm{H}$, Menasche R. Pesquisando na interface: Problemas e desafios a partir da pesquisa qualitativa em saúde. Interface 2014;18(50):449-56. DOI: $10.1590 / 1807-$ $\underline{57622014.0263}$

17- Reis AT, Santos RS, Paschoal Júnior A, Araújo GF. A escuta atenta: Reflexões para a enfermagem no uso do método história de vida. Rev Min Enferm. 2012 [citado em 10 out 2018]; 16(4):617-22. Disponível em: www.reme.org.br/artigo/detalhes/569

18- Parreira PM, Pereira FC, Arreguy-Sena C, Salgueiro A, Tosoli AMG, Marques SC, et al. Social representations of entrepreneurship in nursing students: The role of education in the acquisition of entrepreneurial skills. Sylwan 2015 [citado em 13 out 2018]; 159:23-6. Disponível em: https://www.researchgate.net/publication/29954 1980 Social representations of entrepreneurshi $p$ The role of training in the acquisition of $e$ ntrepreneurial skills

19- Alba MV. Aproximação conceitual desde a escola vigotskiana à teoria das representações sociais. Psicol Estud. 2016;21(1):17-28. DOI: 10.4025/psicolestud.v21i1.29663

20- Marcolino EC, Dias GA, Miranda FAN, Pinheiro MGC, Clementino FS, Simpson CA. Theory of social representations: Educational possibilities in the development of active methodologies for significant learning. Int Arch Med. 2016;9(289):1-7. DOI: 10.3823/2160

21- Crossetti MGO. Revisão integrativa de pesquisa na enfermagem: $O$ rigor científico que Ihe é exigido. Rev Gaúch Enferm. 2012;33(2):8-9. DOI: $10.1590 / \mathrm{S} 1983-14472012000200001$

22- Fernandes ID, Teixeira GAS, Silva MG, Florêncio RMS, Silva RMO, Santa Rosa DO. Expansão da educação superior no Brasil: Ampliação dos cursos de graduação em enfermagem. Rev Latino-Am Enfermagem 2013 [citado em 20 out 2018]; 21(3):670-8. Available in: http://www.scielo.br/pdf/rlae/v21n3/pt 01041169-rlae-21-03-0670.pdf

23- Coelho EAC, Nascimento ER, Paiva MS. Novos saberes, novas perspectivas: 10 anos do Curso de doutorado em enfermagem na UFBA. Rev Baiana Enferm. 2016; 30(1):292-4. DOI: 10.18471/rbe.v1i1.16264

24- Scochi CGS, Gelbcke FL, Ferreira MA, Lima MADS, Padilha KG, Padovani NA, et al. Doutorado em Enfermagem no Brasil: Formação em pesquisa e produção de teses. Rev Latino-Am Enfermagem 2015; 23(3):387-94. DOI: 10.1590/0104$\underline{1169.0590 .2564}$

Nota: Elaborado a partir da disciplina Teoria das Representações Sociais, ministrada pelo Programa de PósGraduação em Enfermagem da Universidade Federal do Rio Grande do Norte.

Recebido em: 01/04/2019

Aprovado em: 15/10/2019

Endereço de correspondência:

Mônica Gisele Costa Pinheiro

Rua Nossa senhora de Lourdes, 125. CEP: 59015-260 - Natal/RN - Brasil

E- mail: monicapinheiro @live.com 